\title{
Genomic Analysis of Prophages Recovered from Listeria monocytogenes Lysogens Found in Seafood and Seafood-Related Environment
}

\author{
Hue Thi Kim Vu ${ }^{1,2}\left(\mathbb{D}\right.$, Matthew J. Stasiewicz ${ }^{3}(\mathbb{D})$, Soottawat Benjakul ${ }^{4}(\mathbb{D})$ and Kitiya Vongkamjan ${ }^{1,4,5, *(\mathbb{D})}$ \\ 1 Department of Food Technology, Prince of Songkla University, Hat Yai, Songkhla 90112, Thailand; \\ kimhue300887@gmail.com \\ 2 Department of Veterinary Hygiene and Food Safety, National Institute of Veterinary Research (NIVR), \\ Dong Da, Hanoi 11519, Vietnam \\ 3 Department of Food Science and Human Nutrition, University of Illinois at Urbana-Champaign, \\ Urbana, IL 61801, USA; mstasie@illinois.edu \\ 4 International Center of Excellence in Seafood Sciences, Faculty of Agro-Industry, Prince of Songkla University, \\ Hat Yai, Songkhla 90112, Thailand; soottawat.b@psu.ac.th \\ 5 Department of Biotechnology, Faculty of Agro-Industry, Kasetsart University, Chatuchak, \\ Bangkok 10900, Thailand \\ * Correspondence: kitiyavongkamjan.a@ku.th
}

check for updates

Citation: Vu, H.T.K.; Stasiewicz, M.J.; Benjakul, S.; Vongkamjan, K. Genomic Analysis of Prophages Recovered from Listeria monocytogenes Lysogens Found in Seafood and Seafood-Related Environment. Microorganisms 2021, 9, 1354. https://doi.org/10.3390/ microorganisms 9071354

Academic Editor: Bruce S. Seal

Received: 14 April 2021

Accepted: 19 June 2021

Published: 22 June 2021

Publisher's Note: MDPI stays neutral with regard to jurisdictional claims in published maps and institutional affiliations.

Copyright: (c) 2021 by the authors. Licensee MDPI, Basel, Switzerland. This article is an open access article distributed under the terms and conditions of the Creative Commons Attribution (CC BY) license (https:// creativecommons.org/licenses/by/ $4.0 /)$.

\begin{abstract}
A prophage is a phage-related sequence that is integrated into a bacterial chromosome. Prophages play an important role in bacterial evolution, survival, and persistence. To understand the impact of Listeria prophages on their host genome organizations, this work sequenced two L. monocytogenes strains (134LM and 036LM), previously identified as lysogens by mitomycin C induction. Draft genomes were generated with assembly sizes of 2,953,877 bp and 3,000,399 bp. One intact prophage $(39,532 \mathrm{bp})$ was inserted into the comK gene of the 134LM genome. Two intact prophages (48,684 bp and 39,488 bp) were inserted in tRNA-Lys and elongation-factor genes of the 036LM genome. The findings confirmed the presence of three corresponding induced phages previously obtained by mitomycin $C$ induction. Comparative genomic analysis of three prophages obtained in the newly sequenced lysogens with 61 prophages found in L. monocytogenes genomes, available in public databases, identified six major clusters using whole genome-based phylogenetic analysis. The results of the comparative genomic analysis of the prophage sequences provides knowledge about the diversity of Listeria prophages and their distribution among Listeria genomes in diverse environments, including different sources or geographical regions. In addition, the prophage sequences and their insertion sites contribute to the genomic diversity of L. monocytogenes genomes. These data of prophage sequences, prophage insertion sites, and prophage sequence comparisons, together with ANIb confirmation, could be useful for L. monocytogenes classification by prophages. One potential development could be refinement of prophage typing tools for monitoring or surveillance of L. monocytogenes contamination and transmission.
\end{abstract}

Keywords: comparative genomic analysis; L. monocytogenes lysogen; Listeria prophage; phage sequencing analysis

\section{Introduction}

L. monocytogenes is a foodborne pathogen and the causative agent of listeriosis, a potentially fatal infection with a mortality rate up to $30 \%[1,2]$. A number of listeriosis outbreaks have been linked to contamination of L. monocytogenes in a variety of foods [3,4], including seafood. L. monocytogenes is well adapted to survive in stressful environments [5-7], which enhance persistence in food associated environments [8].

Comparative genomic analysis of L. monocytogenes isolates from food environments previously showed that prophages play an important role in the evolution of L. mono- 
cytogenes [9], facilitating the survival and persistence of this pathogen over time [10-12]. Prophages could contribute to the genetic variation of Listeria lysogens (prophage-carrying Listeria) $[13,14]$. They are commonly found in the genomes of L. monocytogenes. Multiple prophages, or monocins (an incomplete phage particle derived from a cryptic prophage), were observed in multiple strains, such as F6854, L99, HCC23, J0161 [9,13]. In addition, previous studies have also shown that prophages could serve as epidemiological markers for L. monocytogenes [15]. Therefore, the study of prophage diversity contributes to understanding bacterial genetic variation [16].

Several temperate Listeria phages have been sequenced [17-19]. They showed a basic genome organization comprising of: (i) DNA packaging; (ii) head structural component and assembly; (iii) tail and base plate; (iv) cell lysis; (v) lysogeny control; and (vi) DNA replication, recombination, modification, and gene expression. Most previously sequenced temperate phages were isolated from various natural environments, including phages LP-030-2 and LP-101 from dairy farm samples [20], and phages vB_LmoS_188 and vB_LmoS_293 from mushroom and mushroom compost [21]. In addition, genomes of three Listeria phages induced from the lysogens found in food and food-related environment were sequenced in a previous study [22]. The sequences of these induced phages were carried out in this study to compare with sequences of other known temperate Listeria phages.

Specifically, genomes of two selected L. monocytogenes lysogens isolated from seafood and a seafood-related environment were sequenced to explore the prophage regions or any potential reorganization, compared to the genome sequences of corresponding induced phages. Potential insertion sites and attachment sites were predicted to confirm the prophage border in the host Listeria genomes. Analysis of the prophage sequences was performed with sequences of their corresponding induced phages and those publicly available. Overall, information obtained here allows us to better understand (i) the relationship between prophages and prophage-related regions in the genomes of L. monocytogenes and (ii) the genomic diversity of temperate Listeria phages from different environments or various geographical regions.

\section{Materials and Methods}

\subsection{Selection of Representative L. monocytogenes Lysogens for Genome Sequencing}

To understand the prophage content and their organization in the L. monocytogenes lysogens, 2 isolates (PSU-KV-134LM and PSU-KV-036LM, hereafter identified as 134LM and 036LM, respectively) were selected for sequencing. These two lysogens were isolated from different sources, including a seafood product (fish stick) by Vongkamjan et al. (2016) [23] and a seafood processing environment by Vongkamjan et al. (2015) [24]. The L. monocytogenes lysogen 134LM harbors one inducible prophage and the L. monocytogenes lysogen 036LM harbors two inducible prophages [25].

\subsection{DNA Extraction and Library Preparation for Sequencing}

A single colony of each Listeria isolate was inoculated in $10 \mathrm{~mL}$ of Brain Heart Infusion (BHI, Oxoid, UK) broth and incubated at $30^{\circ} \mathrm{C}$ with shaking (220 rpm) for $16 \mathrm{~h}$, followed by a centrifugation at $6000 \mathrm{rpm}$ for one minute before cell harvesting. Then, bacterial DNA was extracted using GF-1 bacterial DNA extraction kit (Vivantis, Selangor Darul Ehsan, Malaysia) following the manufacturer's instruction. DNA quality and concentration were measured with a Maestro Nano Drop Spectrophotometer (Green bioresearch, LA, USA). The extracted DNA was subsequently used for sequencing library preparation by random fragmentation of the DNA sample using Illumina TruSeq DNA PCR-free kit, followed by $5^{\prime}$ and $3^{\prime}$ adapter ligation. Then, the high-quality sequencing fragmentation libraries were sequenced using the Illumina HiSeq2500 platform with 100-bp paired-end reads at Macrogen Inc. (Seoul, Korea). 


\subsection{Genome Assembly and Annotation of L. monocytogenes Lysogens}

After sequencing, raw reads was filtered to remove the low-quality reads using Trimmomatic [26], then de novo genome assembly was performed using SOAPdenovo2 [27]. Genome sequence of L. monocytogenes F2365 (GenBank accession no. NC002973.6) was used as a reference genome for alignment and ordering contigs using MAUVE genome alignment version 2.4.0 [28]. Subsequently, automatic annotation was performed with the draft genomes using RAST [29]. Genomes were then submitted to the NCBI Prokaryotic Genomes Automatic Annotation Pipeline (PGAAP) (http:/ / www. ncbi.nlm.nih.gov/genomes/static/Pipeline.html, accessed date 13 July 2018) before receiving the GenBank accession numbers. tRNAscan-SE [30] was used to predict tRNA genes. RNAmmer [31] was used to predict rRNA genes. In addition, genomes of the two $L$. monocytogenes lysogens were submitted to the Center for Genomic Epidemiology website (https: / / cge.cbs.dtu.dk/services/MLST/, accessed on 1 June 2021) MLST tool for MLST sequence typing [32].

For these two sequences, and any L. monocytogenes strains used below without the following information, the corresponding clonal complex (CC) and Lineage were defined in BIGSdb via links to the PubMLST website (https: / pubmlst.org/data/profiles / lmonocytogenes.txt, accessed on 1 June 2021) [33,34]. Serotype group, 'serogroup', was determined based on the core genome MLST (cgMLST) allelic profiles [35]. Moreover, to evaluate the similarities between genomes of L. monocytogenes newly sequenced here and those previously sequenced, the average nucleotide identity based on BLAST+ (ANIb) was calculated using the JSpeciesWS webserver (http://jspecies.ribohost.com/jspeciesws/, accessed on 1 June 2021) [36].

\subsection{Identification and Annotation of Prophages and Prophage-Related Regions in the Newly Sequenced Lysogenic L. monocytogenes Genomes}

The draft genome of each Listeria isolate was submitted to PHASTER for identification of prophages and prophage-related regions [37]. This tool classifies the detected prophage regions as intact, questionable, or incomplete prophage, based on a total score obtained by the completeness of required genes to fully form an assembled virus. The "questionable" or "incomplete prophages" were reported as putatively defective prophage as lacks of many structural phage genes. Thus, these putatively defective prophages were exclude from our analysis. The intact prophage was confirmed by identifying the presence of the phage integrase gene (int) that was adjacent to a potential bacterial gene, which could be a potential insertion site. To identify a complete sequence of each identified intact prophage, full gene clusters of prophages [18] were considered together with the potential insertion site.

Each complete sequence of the intact prophage was extracted for comparative analysis starting from $3^{\prime}$ end of the corresponding phage integrase gene through the $5^{\prime}$ end of the last phage genes detected by PHASTER. Generally, this site was several genes after a phage endolysin gene. PHASTER also detects a prophage attachment (att) site by searching for a sequence region with short nucleotide repeats (12-80 nucleotides) in both left and right junction fragments (attL and attR).

Then, the extracted intact prophage sequence was submitted for annotation using RAST [29]. Annotation results from PHASTER [37] were also used. The annotations were curated and verified using BLAST [38] and Artemis [39]. Functional domains were predicted using InterPro (http:/ / www.ebi.ac.uk/interpro, accessed on 1 June 2021) [40]. In addition, tRNA genes were predicted using tRNAscan-SE webserver [30].

\subsection{Variation of Prophages Inserted in Multiple Loci of L. monocytogenes Genomes}

A total of 163 L. monocytogenes strains with complete genomes available on the National Center for Biotechnology Information (NCBI) database were subjected to prophage screening by PHASTER (http:/ / phaster.ca/, accessed on 1 June 2021) with default settings. "Intact prophage" were manually confirmed as the prophages by the presence of 
the attachment site (att), integrase gene, terminases, and structural viral proteins. All 163 L. monocytogenes strains were subjected to MLST sequence typing (ST) [32]. ST and prophage presence were used to select $35 \mathrm{~L}$. monocytogenes strains for allow up analysis (Table 1). The 14 STs of selected L. monocytogenes strains have global distribution [41-43]. Further, the 10 L. monocytogenes of ST1 are of ST overrepresented in clinical and food isolates worldwide [44]. The information of L. monocytogenes sequence type (global distribution and represented in clinical and food isolates) and prophage complement (having complete prophage sequence(s) and identified attachment site(s)) were used in parallel for the selection of L. monocytogenes strains to be included in our study.

All of these detected intact prophages obtained from 35 L. monocytogenes genomes were submitted to PHASTER [37] for prophage prediction and confirmation. For each detected intact prophage, prophage insertion site and prophage borders were manually validated (as described above) and showed that containing prophage(s) with previously reported insertion sites, including two comK-prophages, ten tRNA-Lys prophages, tRNAArg, tRNA-Ser, tRNA-Thr, elongation factor gene, and/or ribosomal protein S9.

For comparative genomic analysis of prophages, the sequences of three obtained prophages in the two newly sequenced lysogens and this database set were included. Overall comparison of 64 prophage sequences was conducted using intergenomic distances inferred with the Genome-Blast Distance Phylogeny (GBDP) tool [45] using settings recommended for prokaryotic viruses. The intergenomic distances were used to infer a balanced minimum evolution tree with branch support from 100 pseudo-bootstrap replicates. A phylogenetic tree of the prophage sequence alignment was generated using VICTOR with default settings and visualized by FigTree v1.4.3 [46]. Linear genome visualizations of prophages within each identified cluster were generated using Easyfig 2.1 [47]. BLASTn was used to determine the nucleotide similarities of the prophage sequences found in two L. monocytogenes lysogens newly sequenced and the closely related prophages [38].

\subsection{Comparison of Prophages Present in the Lysogenic Genomes and the Corresponding Induced Phages}

Comparative genomic analysis was performed using sequences of the three prophages (pp-134LM-comK, pp-036LM-EF, and pp-036LM-tRNA-Lys) found in the genomes of the two L. monocytogenes lysogens sequenced in this study and three Listeria phages (LP019, LP040, and LP041) that were sequenced previously [22]. Induction by Mitomycin C have previously yielded one induced phage, LP019, from the isolate 134LM and two induced phages, LP040 and LP041, from the isolate 036LM [25]. Linear genome visualizations between each pair (prophage sequence and induced phage sequence) was conducted using Easyfig 2.1 [47]. The regions presenting differences between the prophage sequences and the induced Listeria phage sequences were extracted for nucleotide sequence alignment using Clustal Omega [48]. 
Table 1. L. monocytogenes genome sequences presenting prophages and prophage-related regions.

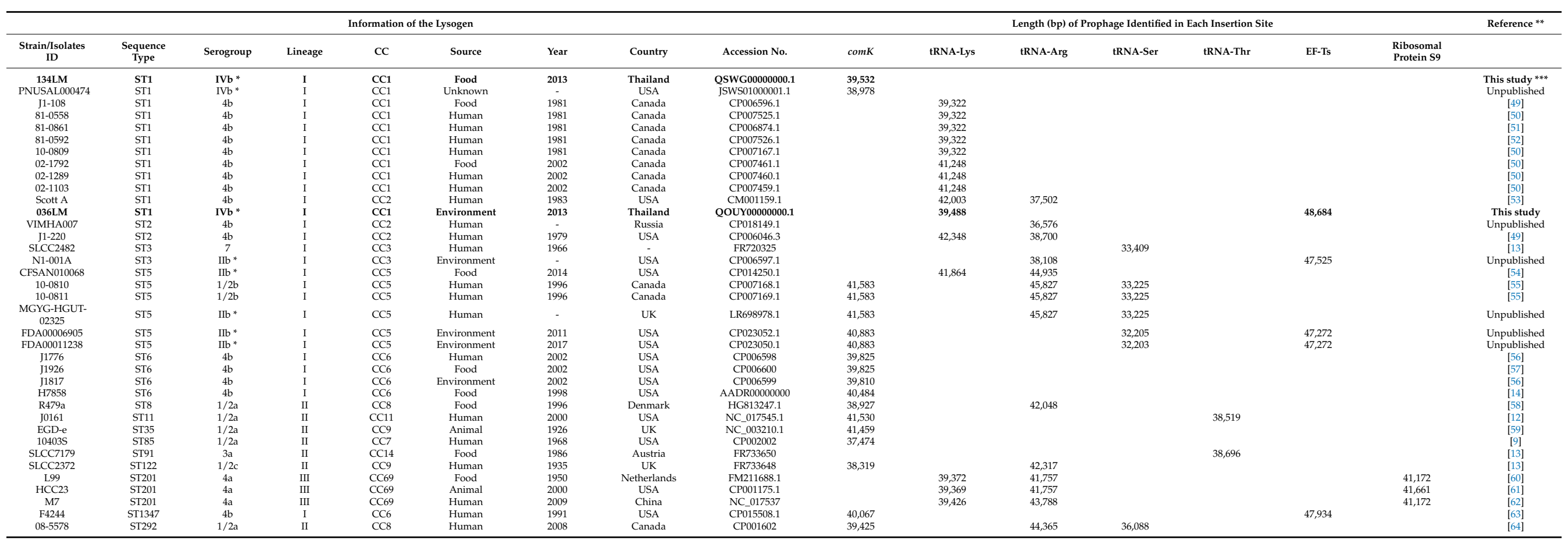

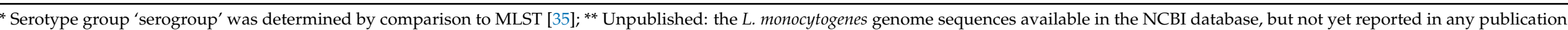
$* * *$ L. monocytogenes lysogens sequenced in this study are bolded. 
2.7. Comparative Genomic Analysis of the Newly Sequenced Listeria Phages and the Previously Sequenced Temperate Listeria Phages

Full genome sequences of all temperate Listeria phages available on the NCBI database were obtained (Table 2).

Table 2. Temperate Listeria phage genome sequences used for comparative analysis.

\begin{tabular}{cccc}
\hline Temperate Listeria Phage & Genome Size (bp) & NCBI Accession No. & Reference \\
\hline LP019 & 38,601 & MH341451 & {$[22]$} \\
LP040 & 39,585 & MH341452 & {$[22]$} \\
LP041 & 48,286 & MH341453 & {$[22]$} \\
A118 & 40,834 & NC_003216.1 & {$[18]$} \\
A006 & 38,124 & NC_009815.1 & {$[19]$} \\
B025 & 42,653 & NC_009812.1 & {$[19]$} \\
B054 & 48,172 & NC_009813.1 & {$[19]$} \\
A500 & 38,867 & NC_009810.1 & {$[19]$} \\
PSA & 37,618 & AJ312240.2 & {$[17]$} \\
LP-101 & 43,767 & NC_024387.1 & {$[20]$} \\
LP-030-2 & 38,275 & JX120799.2 & {$[20]$} \\
LP-030-3 & 41,156 & NC_024384.1 & {$[20]$} \\
vB_LmoS_188 & 38,392 & KP399677 & {$[21]$} \\
vB_LmoS_293 & 40,759 & KP399678 & {$[21]$} \\
\hline
\end{tabular}

The whole genome comparison of these temperate phages and our newly sequenced phages was conducted using intergenomic distances inferred with the GBDP tool [45] under settings recommended for prokaryotic viruses. The intergenomic distances were used to infer a balanced minimum evolution tree with branch support from 100 pseudo-bootstrap replicates. A phylogenetic tree of the alignment was generated using VICTOR with default settings and visualized by FigTree v1.4.3 [46]. Nucleotide similarity of each newly sequenced Listeria phages and the closely related temperate Listeria phages was obtained by BLASTn [38]. Linear genome visualizations were generated using Easyfig 2.1 [47].

\subsection{Nucleotide Sequence Accession Numbers}

Sequences of L. monocytogenes lysogens 134M and 036LM were deposited at DDBJ/ENA/ GenBank under the accession numbers QSWG00000000 and QOUY00000000, respectively. The versions described in this paper are QSWG01000000 and QOUY01000000. FASTQ raw sequence files for L. monocytogenes lysogens 134M and 036LM have been deposited in the Sequence Read Archive (SRA) database at NCBI with SRA accession number SRP162378 and SRP163030 under BioProject PRJNA484544 and PRJNA480937, respectively.

\section{Results}

\subsection{Draft Genomes of Lysogenic L. monocytogenes}

The draft genomes of L. monocytogenes 134LM and 036LM yielded 21 and 19 million reads with an estimated coverage of $715 \times$ and $643 \times$, respectively (Table 3 ). Genome assembly size of L. monocytogenes 134LM was 2,953,877 bp with 2955 detected CDSs (11 contigs). Assembly size of L. monocytogenes 036LM was 3,000,399 bp with 3027 detected CDSs (11 contigs). The GC content of both $L$. monocytogenes lysogens was $37.8 \%$. A total of 54 tRNAs and four complete rRNA operons (16S-23S-5S) were found in 134LM genome, while 52 tRNAs and three complete rRNA operons were found in 036LM genome. The genomes of these two lysogens showed $99.94 \%$ sequence similarity as observed by ANIb (Supplement Table S1), and shared 99.99\% similarity to the genome of L. monocytogenes reference strain F2365. In addition, genomes of the two L. monocytogenes lysogens were classified into MLST sequence type 1 (CC1), lineage I, and belonged to L. monocytogenes serogroup IVb (which includes serotypes 4b, 4ab, 4d, and 4e). 
Table 3. Lysogenic L. monocytogenes draft genome information.

\begin{tabular}{ccc}
\hline Description & PSU-KV-134LM (134LM) & PSU-KV-036LM (036LM) \\
\hline No. of reads (pre-filtered) & $27,525,582$ & $24,257,104$ \\
No. of reads (post filtered) & $21,137,912$ & $19,286,526$ \\
Assembly size (bp) & $2,953,877$ & $3,000,399$ \\
Sequencing coverage & $715 \times$ & $643 \times$ \\
GC content (\%) & 37.80 & 37.83 \\
Number of contigs & 11 & 11 \\
Shortest contig size (bp) & 1778 & 3570 \\
Longest contig size (bp) & 766,006 & $1,291,185$ \\
N50 value & 476,941 & 556,523 \\
No. of predicted CDSs & 2955 & 3027 \\
No. of unique rRNAs (5S, 16S, 23S) & $2,1,1$ & $1,1,1$ \\
No. of tRNAs & 54 & 52 \\
No. of prophage regions & 2 & 3 \\
Length of intact prophage (bp) & 39,532 & 48,684 and 39,488 \\
Length of questionable prophage (bp) & 10,729 & 10,729 \\
\hline
\end{tabular}

3.2. Identification and Annotation of Prophages and Prophage-Related Regions in the Genomes of Lysogenic L. monocytogenes Newly Sequenced

PHASTER program identified one intact prophage in the 134LM genome (pp-134LMcomK; 39,532 bp) and two intact prophages in the 036LM genome (pp-036LM-EF; 48,684 bp and pp-036LM-tRNA-Lys; 39,488 bp) (Table 3 and Figure 1). In addition, a conserved questionable prophage $(10,729 \mathrm{bp})$ was found in both genomes. The major differences between the two lysogenic genomes were the inserted prophage sequences: $90 \mathrm{~kb}$ inserted in the genome of 036LM and $40 \mathrm{~kb}$ inserted in the genome of 134LM. These prophages were found in different insertion sites. Those with a comK-prophage insertion resulted in a separation of the comK gene. However, prophage pp-036LM-EF was inserted in a translation elongation factor gene (EF) and prophage pp-036LM-tRNA-Lys was located at the 3'-end of tRNA-Lys of the 036LM genome. Various repeat nucleotide sequences as phage attachment sites were identified: (i) "ATGATTATAATAAT" for prophage pp-134LM-comK, (ii) "TGATAACAAAGC" for prophage pp-036LM-EF and (iii) "ACTCTTAATCAGCGGGTCGGGGGTTCGAAACCCTCACAACCCATA" for prophage pp-036LM-tRNA-Lys.
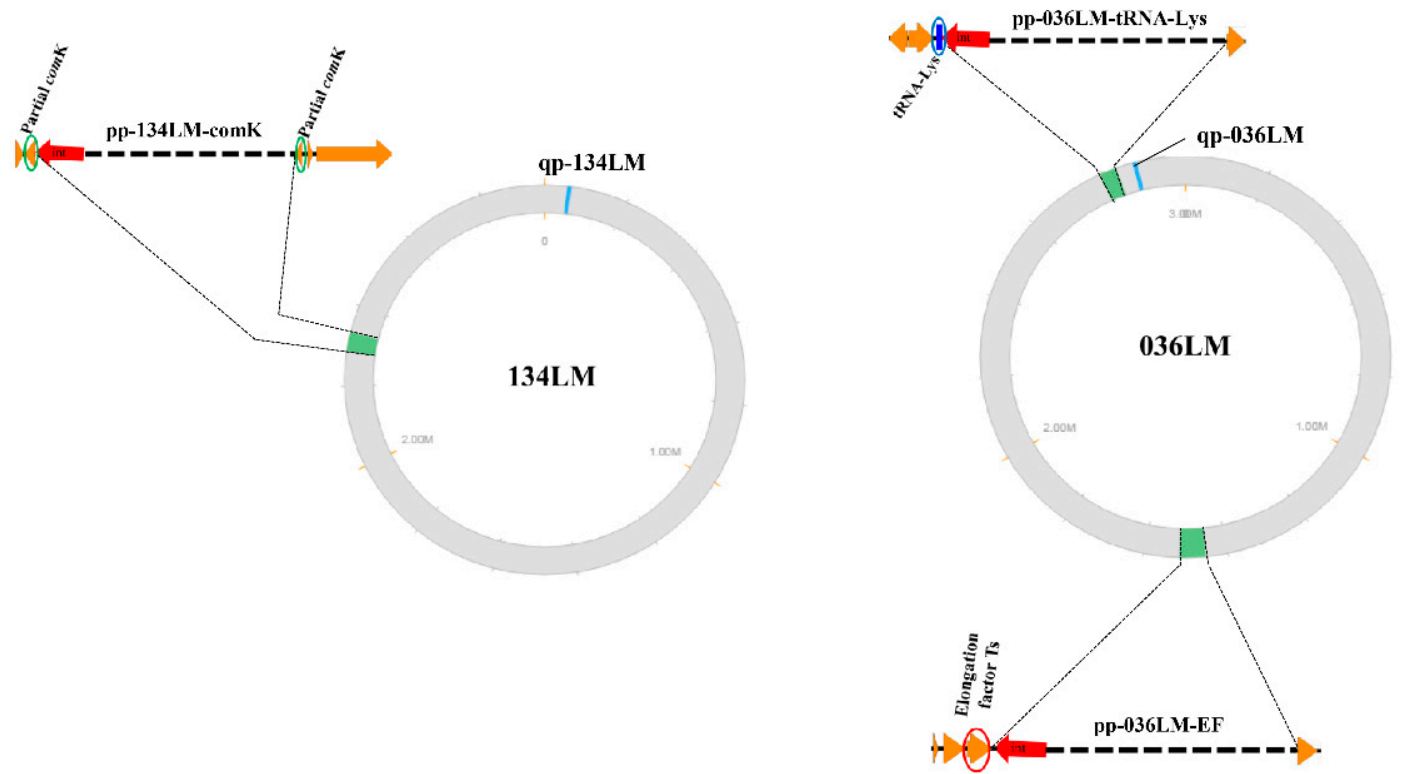

Figure 1. Locations of the intact prophages (pp) and questionable prophage (qp) detected in 134LM/036LM genomes.

The GC content of prophage pp-134LM-comK and pp-036LM-tRNA-Lys was 37.8\%, while GC content of prophage pp-036LM-EF was $37.1 \%$. Genome annotation of three 
prophages (Figure 2) revealed that prophage pp-134LM-comK contained 68 ORFs, of which 29 ORFs were assigned functions. Prophage pp-036LM-EF contained 67 ORFs, of which 32 ORFs were assigned functions. Prophage pp-036LM-tRNA-Lys contained 81 ORFs, of which 23 ORFs were assigned functions. Overall, the annotation of all three prophage sequences contained six main gene clusters: (i) lysogeny control; (ii) DNA replication, recombination, modification and gene expression; (iii) DNA packaging; (iv) head structural component and assembly; (v) tail and base plate; and (vi) cell lysis. In addition, the questionable prophage identified in both L. monocytogenes genomes, has been previously reported as a monocin $[60,65]$. These elements harbored a complete $\operatorname{lmaDCBA}$ operon identified as a virulence factor of L. monocytogenes.
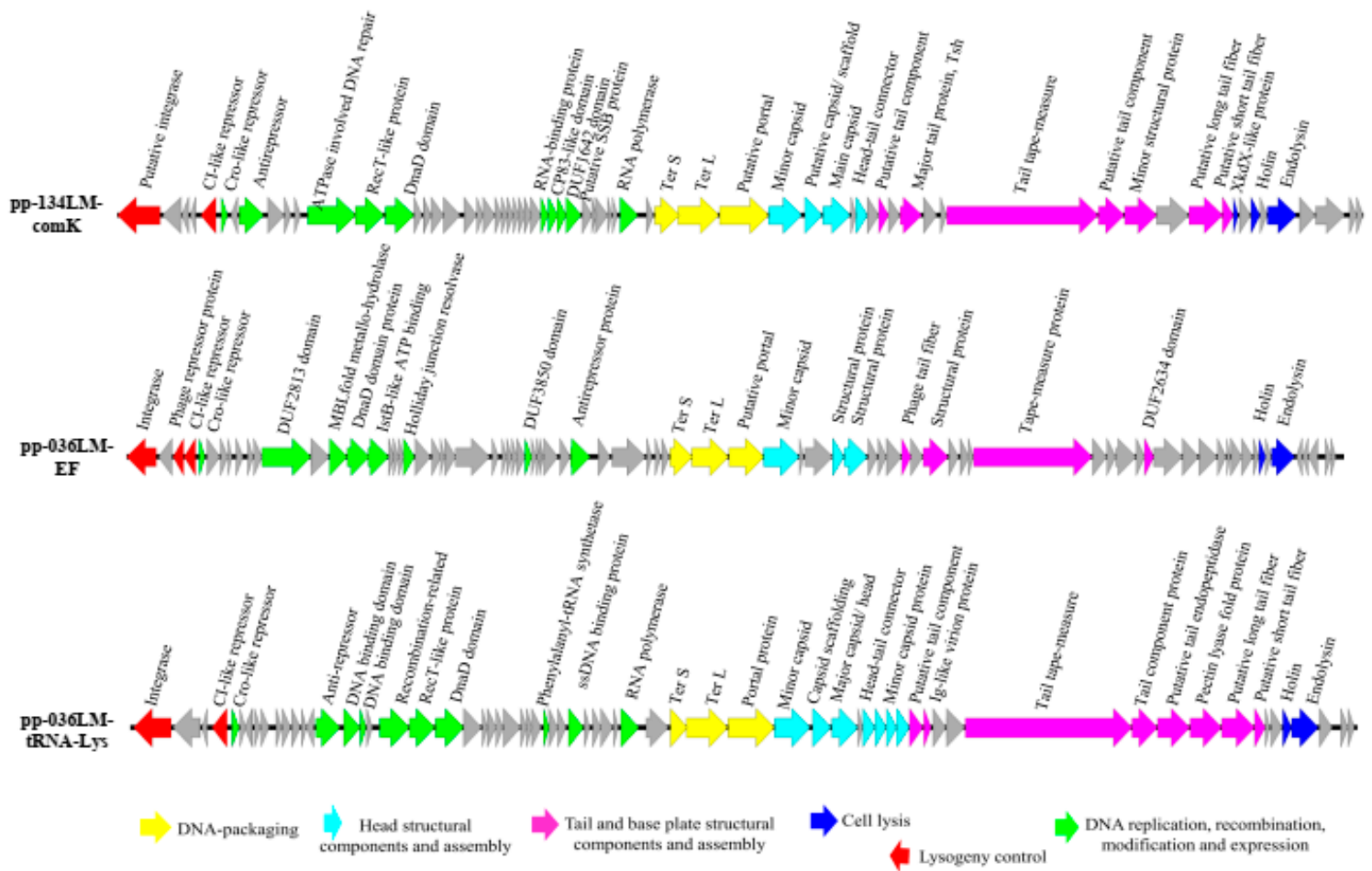

Figure 2. Genome annotation of prophage sequences present in the genomes of L. monocytogenes 134LM and 036LM.

\subsection{Sequence Comparison of Prophages and Their Corresponding Induced Phages}

Overall, sequence comparison of prophages and the corresponding induced phages showed similar genes and gene functions (Figure 3). However, genome organization of the prophage was different from the induced phages. Specifically, the lysogeny control cluster and the induced phage early genes, which encoded for products of DNA replication, recombination, modification, and expression were located in the downstream of the prophage sequences. In contrast, the induced phage late genes encoding for products of DNA packaging, head, tail, and base plate structural and assembly proteins, were in the upstream of the prophage sequences. The phage integrase gene is always arranged in the downstream of prophage genome.

Several sequence variations were observed regions (labeled i to iv in Figure 3) of the prophage sequences. For example, an additional fragment of $1028 \mathrm{bp}$ was present in the sequence of prophage pp-134LM-comK as compared to its corresponding induced phage LP019 sequence. This fragment encodes for hypothetical proteins ORF52-ORF55 of Listeria phage vB_LmoS_293. A 55-bp-fragment with some nucleotide modifications was found in the sequence of prophage pp-036LM-tRNA-Lys, thus differentiating this from the sequence of corresponding induced phage LP040. This could be a single/multiple nucleotide polymorphisms changed between the two sequences (induced phage and integrated prophage). 


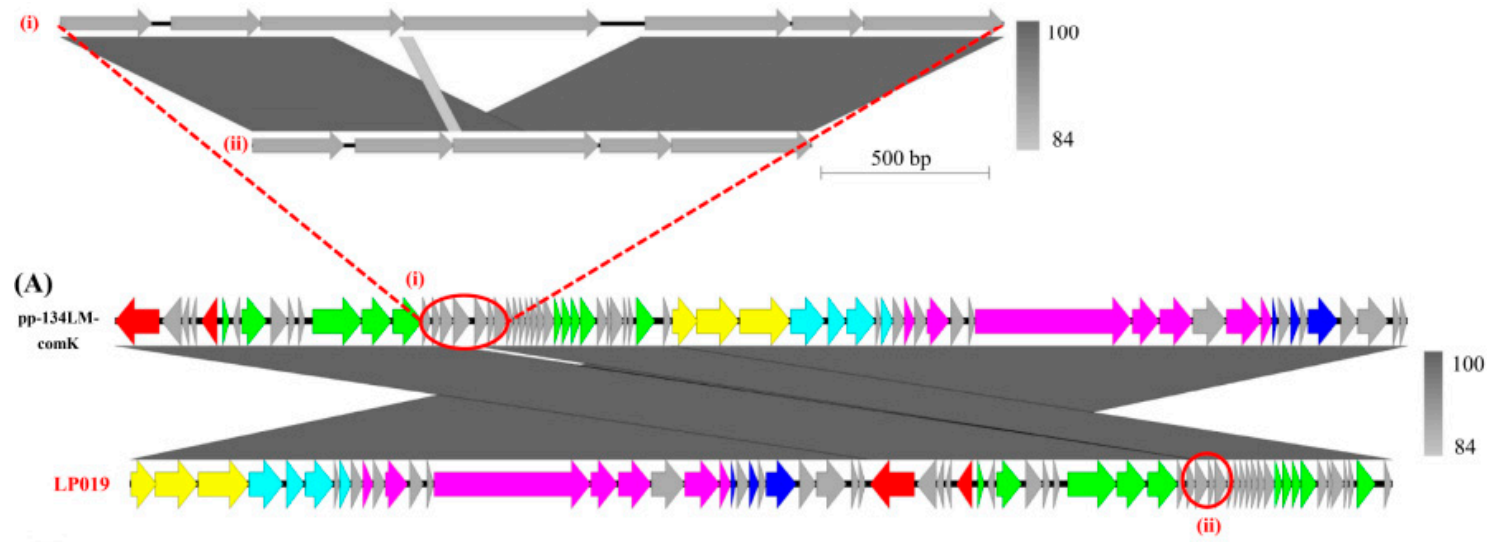

(1)
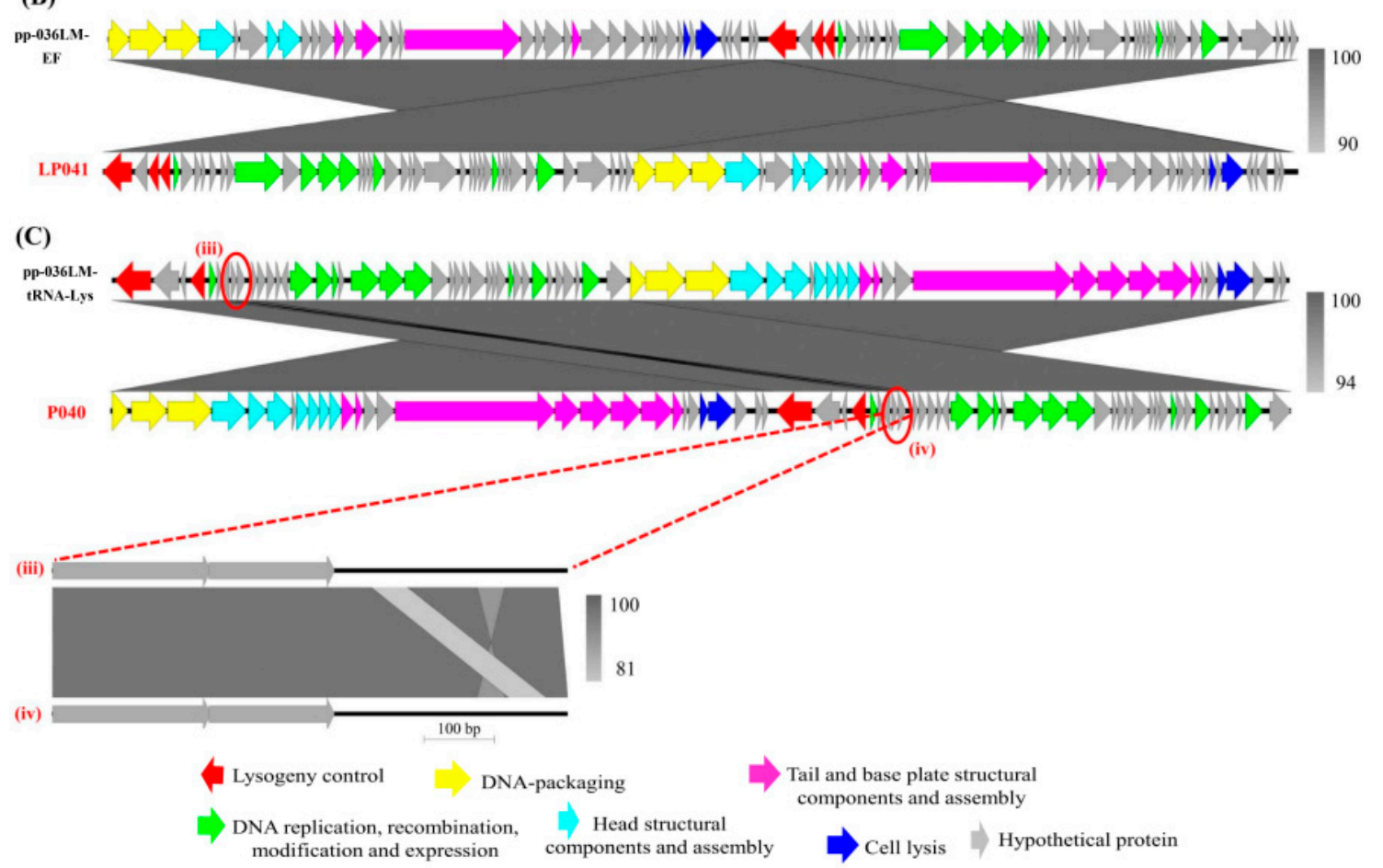

Figure 3. Genome comparison of the prophage sequences and their corresponding induced Listeria phages: (A) prophage pp-134LM-comK and phage LP019; (B) prophage pp-036LM-EF and phage LP041; (C) prophage pp-036LM-tRNA-Lys and phage LP040. The zoomed panel indicates variations in the sequences of prophage and the corresponding induced phage. The shade of grey between genomes indicates the level of nucleotide similarity (darker is higher similarity).

\subsection{Prophages Inserted in Multiple Loci of L. monocytogenes Host Genomes}

A total of 61 intact prophages detected by PHASTER within the genomes of 35 selected L. monocytogenes strains and an additional three prophages from two lysogens newly sequenced here were used for further analysis. Overall, these prophages were inserted into seven different insertion loci of L. monocytogenes genomes (Table 1). Prophages of multiple lysogenic strains showed common insertion sites, such as comK gene $(\mathrm{n}=18)$, tRNA-Lys $(n=15)$ and tRNA-Arg $(n=14)$, followed by tRNA-Ser $(n=7)$, EF-Ts $(n=5)$, Ribosomal protein $S 9(n=3)$, and tRNA-Thr $(n=2)$.

The results reveal that many L. monocytogenes genomes with sequence similarity show a high degree of prophage conservation despite these strains being isolated from different sources, distinct geographical locations, and different times. For example, the strain J1776 (from human), J1926 (from food), and J1817 (from environment), shared 99.99-100\% sequence similarity by ANIb (Supplement Table S1). They also harbored a conserved comKprophage with $100 \%$ nucleotide sequence similarity. The L. monocytogenes strain L99 (from 
food, Netherlands, 1950), HCC23 (from animal, USA, 2000), and M7 (from human, China, 2009) shared $99.99-100 \%$ sequence similarity, and they contained the same set of prophages (including tRNA-Lys prophage with the size of 39,369-39,426 bp, tRNA-Arg prophage with the size of $41,757-41,788 \mathrm{bp}$ and ribosomal protein $S 9$ prophage with the size of 41,172-41,661 bp), with 85-100\% nucleotide sequence similarity as determined by BLASTn. Among 12 strains, L. monocytogenes ST1 included for the analysis, L. monocytogenes lysogens 134LM (food source), and PNUSAL000474 (unknown source) shared 99.94\% sequence similarity by ANIb; they also harbored a highly conserved comK-prophage with $90 \%$ nucleotide sequence similarity.

However, many L. monocytogenes genomes with high sequence similarity contained different prophages. In other words, the prophage sequence and their insertion sites also contribute to the genomic diversity of L. monocytogenes genomes. For instance, although the genome of the lysogenic isolate 134LM (from food, Thailand, 2013) showed high sequence similarity (99.97\%) to the genomes of three L. monocytogenes strains 02-1792, 02-1289, and 021103 (from food/ human, Canada, 2002) as identified by ANIb, prophage pp-134LM-comK was found in a different cluster than prophages of the 3 strains 02-1792, 02-1289 and 02-1103 whose insertion sites were tRNA-Lys gene. Similarly, the L. monocytogenes 036LM (from environment, Thailand, 2013) and five L. monocytogenes strains J1-108, 81-0592, 81-0558, 81-0861 and 10-0809 (from food/human, Canada, 1981) shared 99.71\% sequence similarity by ANIb. These strains contained a highly similar tRNA-Lys prophage by $99 \%$ nucleotide sequence similarity. The differences were from an extra 48,684 bp of the EF-TS-prophage inserted in the genome of the L. monocytogenes 036LM. Another example, an additional tRNA-Arg prophage, increased the distance of L. monocytogenes Scott A sequence (from human, USA, 1983) to three sequences of L. monocytogenes 02-1792, 02-1289, 02-1103 (from food/human, Canada, 2002).

\subsection{Variation of Prophage Sequences Found in L. monocytogenes Genomes}

Full genome comparison of 64 prophage sequences by VICTOR classified those into six distinct clusters, I to VI (Figure 4), supported by a bootstrap value of 100 . Prophages with a single insertion site were often classified together. To specify, 13/14 prophages with the tRNA-Arg insertion site were classified together in cluster I. All five prophages with the EF-Ts insertion site were classified in cluster II. Seven prophages with the tRNA-Ser insertion site were classified in cluster IV. In contrast, only 3 of 15 prophages with the tRNA-Lys insertion site were classified in cluster III. However, prophages with various insertion sites were classified into clusters V and VI. Cluster V was the largest cluster, comprising 23 prophages with multiple insertion sites: $\operatorname{com} K$, tRNA-Lys, tRNA-Thr, and ribosomal protein S9. In addition, cluster VI contained 13 prophages with four insertion sites: $\operatorname{comK}$, tRNA-Lys, tRNA-Arg, and tRNA-Thr.

Linear genome visualizations of prophages were generated using Easyfig 2.1 (Supplement Figure S1). Result shows the overall relatedness of prophages despite various prophage insertion sites. For the clusters I to IV, which have prophages with a single insertion site, the integrase gene and nearby hypothetical proteins showed high similarity, while the genes, which encoded for DNA packaging shows higher diversity. In contrast, the two clusters (V and VI) that contained prophages with multiple insertion sites show no relatedness or very low similarity for the integrase gene and nearby hypothetical proteins. These prophages with different insertion sites were classified together because late genes show close relatedness.

Three prophages found in the two lysogens sequenced in this study were classified into clusters II, V, and VI. Interestingly, these prophage sequences were closely related to prophages of L. monocytogenes strains from various sources. For example, the pp-036LMEF prophage showed high similarity to prophage of L. monocytogenes strains N1-011A, FDA00011238, and FDA00006905 (from environment, USA) and F4244 (from human, USA) by $96 \%$ nucleotide sequence similarity. The prophage pp-036LM-tRNA-Lys showed high similarity to prophage of L. monocytogenes strains J1-108, 81-0592, 81-0558, 81-0861, and 
10-0809 (from food/human, Canada) by 99\% nucleotide sequence similarity. The comKprophage of 134LM was the most closely related to a comK-prophage of L. monocytogenes strains PNUSAL000474 (unknown source, USA) and F4244 (from human, USA) by $90 \%$ nucleotide sequence similarity.

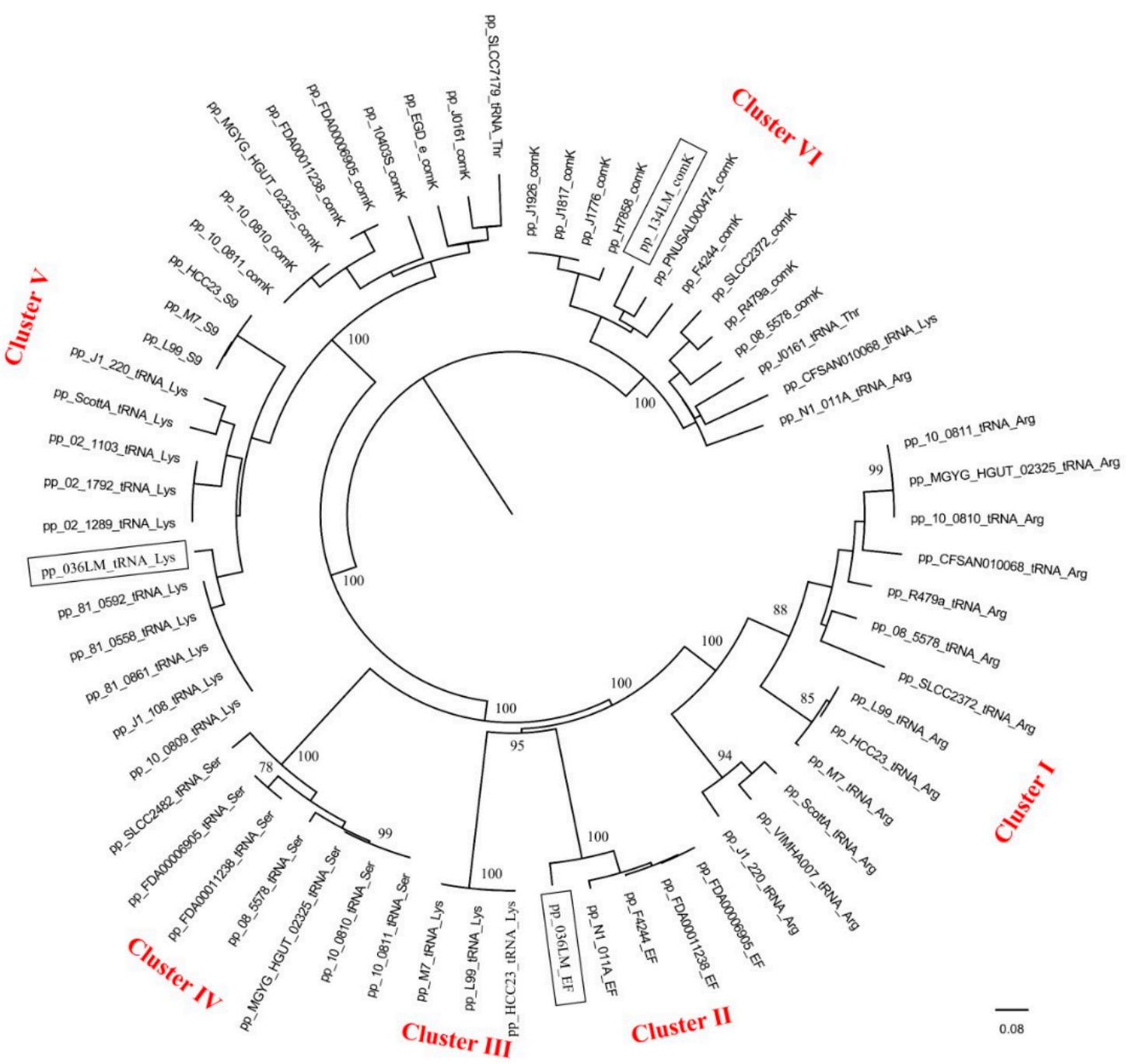

Figure 4. Phylogenetic tree based on full genome comparison of prophage sequences detected in L. monocytogenes genomes. Tree was constructed using whole genome-based phylogenetic analysis. GBDP pseudo-bootstrap support values from 100 replicates are given on the branches if support is $\geq 70 \%$. The scale represents homology $\%$. The three prophage sequences in the genomes of the two L. monocytogenes lysogens sequenced in this study are marked by a square.

\subsection{Comparative Analysis of the Newly Sequenced Induced Phages and the Temperate Listeria Phages from Other Regions}

Genomes of phages sequenced here were closely related to several temperate phages (Figure 5). Phage LP019 was closely related to Listeria phage vB_LmoS_188, which was a temperate phage previously isolated from wild mushroom in Ireland. Phage LP040 was closely related to phage vB_LmoS_293, which was also a temperate phage previously isolated from mushroom compost in Ireland. Phage LP041 was closely related to a unique Listeria phage B054, which was previously induced from L. innocua WSLC 2054 in the U.S. 


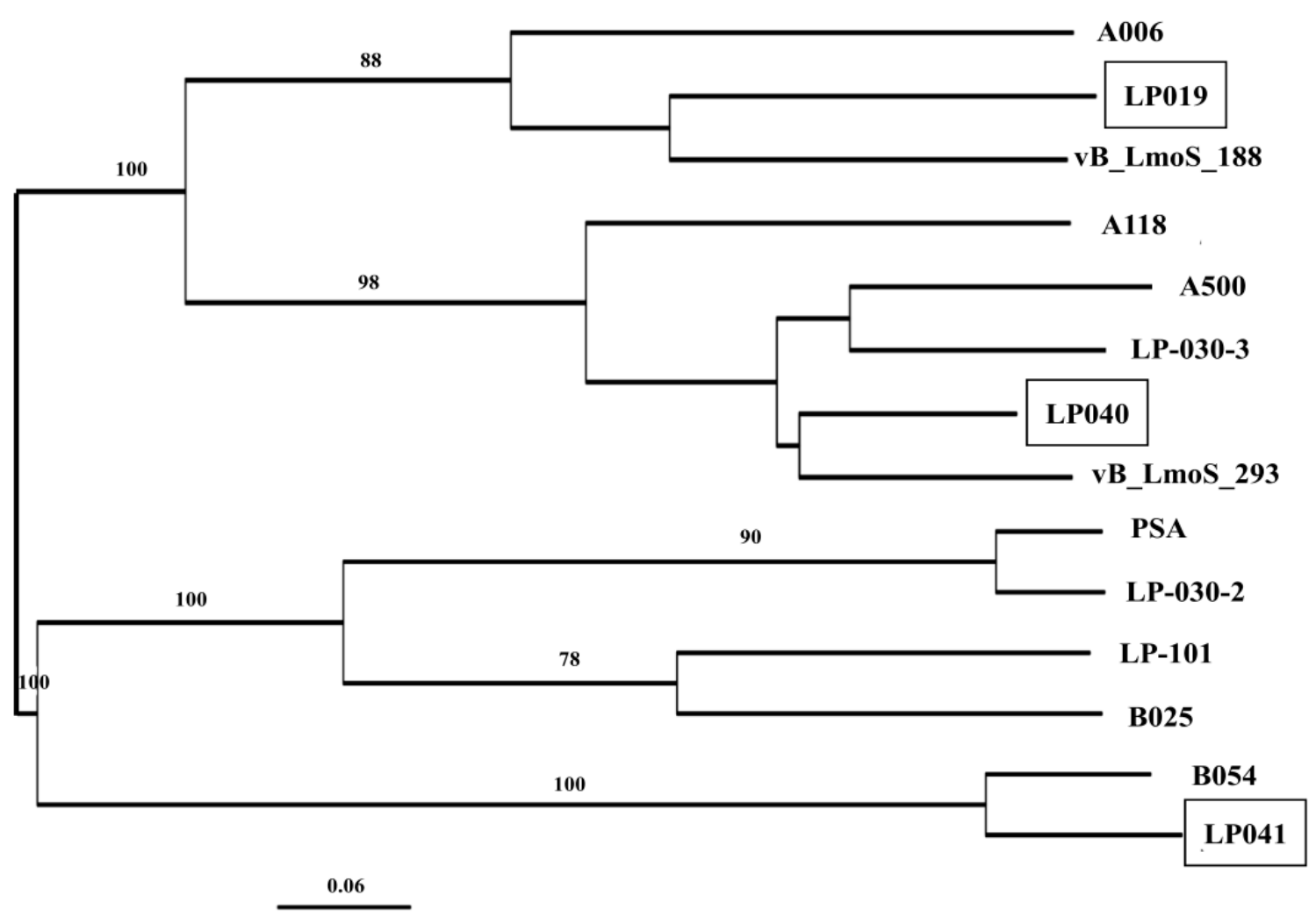

Figure 5. Phylogenetic tree of the newly sequenced phages (in a box) and previously sequenced temperate phages. Tree was constructed using the whole genome-based phylogenetic analysis. GBDP pseudo-bootstrap support values from 100 replicates are given on the branches if support is $\geq 70 \%$.

Genome sequence comparison of each newly sequenced Listeria phage and the closely related phages indicates some regions with high nucleotide sequence similarity $(>90 \%)$, especially the late genes, which encoded for DNA packaging, head, tail, and base plate structure (Figure 6). Overall, partial regions of the lysogeny control cluster and early genes encoded for DNA replication, recombination, modification, and gene expression presented low similarity between each newly sequenced phage, and the closely related phage. However, the integrase gene and nearby hypothetical proteins of phage LP040 and phage LP041 showed high similarity to those of phage vB_LmoS_293 and phage B054, respectively. Interestingly, the Cro/CI repressor and anti-repressor genes involving in the phage genetic switch were found to be less conserved in all the phages analyzed. Sequence comparison of the induced phage LP019 and isolated temperate phage vB_LmoS_188 showed low similarity in most regions, except the late genes. Overall, genomes of our induced phages from seafood and a seafood-related environment were closely related to the temperate phages from natural environments (i.e., wild mushroom, mushroom compost, and dairy farm) or induced phages from lysogens of other Listeria species. 

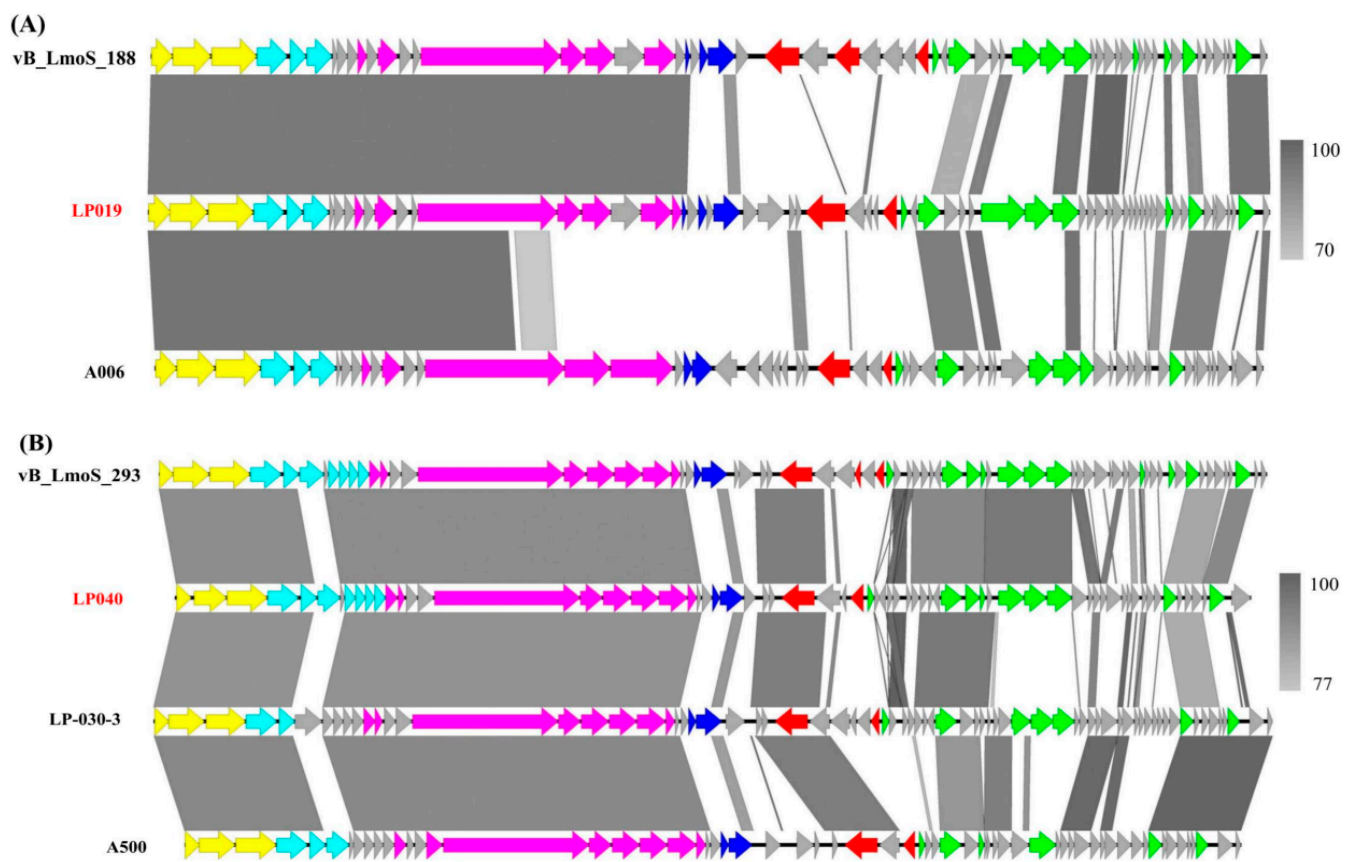

(C)

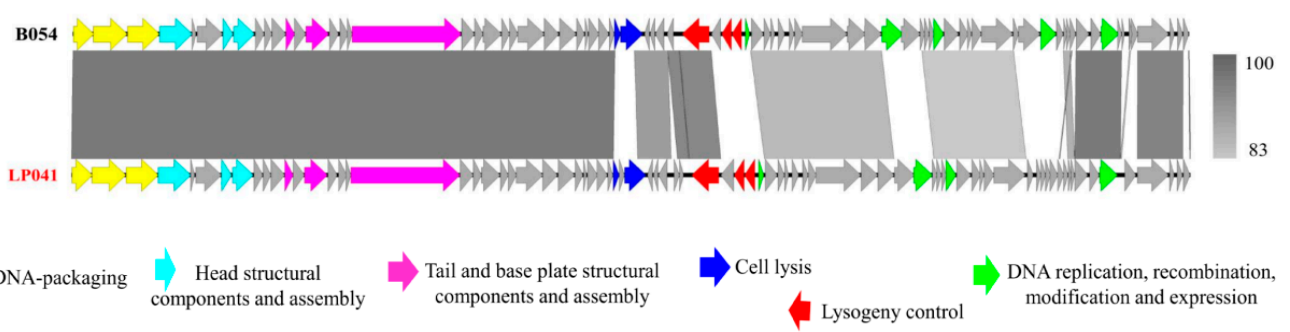

Figure 6. Genome comparison of the newly sequenced phages (performed in previous study [22]) and closely related temperate phages. (A) LP019, (B) LP040, and (C) LP041; predicted genes were indicated by arrows and colored according to major gene functions. The shade of gray drawn between genomes represented the level of nucleotide similarity.

\section{Discussion}

\subsection{Prophages and Prophage-Related Regions Contribute to the Diversity of}

L. monocytogenes Genomes

Overall, the genomes of these lysogens displayed typical genome features of L. monocytogenes such as assembly size $(2.8-3 \mathrm{Mb})$, GC content $(\sim 38 \%)$ as reported previously $[13,66]$. However, the number of unique rRNA operons (16S-23S-5S) found in the genome of 134LM and 036LM were lower than the number of rRNAs of Listeria genomes as commonly reported, which typically showed 13-18 repeat rRNA regions across the chromosome [13,64]. This could be due to the fact the 'repeat' rRNA regions would all map to the same gene when using short read sequencing. Another possible reason for the missing rRNAs is that they could be present in gaps between contigs of the two L. monocytogenes lysogens sequence genomes [44].

The two genomes of lysogenic L. monocytogenes newly sequenced here shared 99.94\% sequence similarity by ANIb. The two intact prophages in the lysogenic genome of 036LM could account for the difference, compared to the genome of 134LM. In addition, genome sequences of both lysogens showed high similarity to the genome of L. monocytogenes reference strain F2365, but prophage was not detected in F2365 and no insertion site was found. Furthermore, the extra EF-Ts prophage in 036LM or the tRNA-Arg prophage in L. monocytogenes Scott A made the difference between these strains and their closely related strains. These findings suggest that prophage sequences could contribute to evolutionary changes of L. monocytogenes genomes. Similarly, Orsi et al. (2008) reported that the differences in 
a 42-kb-prophage sequence differentiate four similar genome backbone L. monocytogenes isolates of a strain which persisted over 12 years, indicating the importance of prophages in differentiation of closely related L. monocytogenes [12].

Prophages were found in different loci of L. monocytogenes genomes as indicated by various insertion sites detected. The comK gene and tRNA-Lys gene were reported as the most common insertion site for Listeria prophages. Previous studies have shown that multiple L. monocytogenes strains such as J0161, J2818, R479a, HCC23 and J1-108 containing comK and/or tRNA-Lys prophage(s) $[9,13]$. Lysogens containing comK-prophages have been previously reported to facilitate the survival over 10 years in foods and food processing environments [11]. The comK-prophage could also help the Listeria lysogen escape from activated macrophages, facilitating the virulence of $L$. monocytogenes when this bacteria infects a human host [67]. This prophage insertion site could therefore enhance the survival and virulence of Listeria lysogen. In addition, the EF gene was another insertion site for Listeria prophages detected in this study. This is considered as a rare insertion site, which has also previously been found in a few strains of L. monocytogenes such as SLCC2755 [13] and L. innocua strain Clip11262 [59]. The EF gene plays an important role in facilitating the translational elongation of protein synthesis of the L. monocytogenes. Overall, prophage insertion loci could be influenced by the integrase gene or the bacterial attachment sites [68].

Full genome comparison showed high sequence similarity by ANIb analysis between L. monocytogenes $134 \mathrm{LM}$ newly sequenced here and L. monocytogenes strains on the database such as 02-1792, 02-1289, and 02-1103. However, the prophage in the genome of L. monocytogenes 134LM showed a different insertion site and was found in a different phylogenetic cluster than those closely related strains. These findings suggest that prophage sequences and the insertion sites could provide additional information for identifying the variations in the genomes of Listeria beyond the analysis by ANIb. Similarly, previous studies have revealed that the genetic diversity of L. monocytogenes was affected by gain or loss of genetic islands, especially the presence of prophage sequences $[9,13,69]$. In addition, L. monocytogenes strains showing prophage with the same genome size), insertion site, and classified into the same cluster, could have relatedness confirmed by ANIb analysis. Therefore, data from the detection of prophages, differentiation of insertion sites in specific loci of Listeria, and prophage sequence comparison, together with the ANIb confirmation, could be used to further classify L. monocytogenes strains.

We found that the prophage insertion sites could be diverse in L. monocytogenes genomes, which contributes to the genomic diversity of L. monocytogenes genomes. Moreover, variation of prophage sequences was found in the genomes of L. monocytogenes, suggesting diverse types of prophages were found in L. monocytogenes genomes, which play an important role in the host genome diversity. Previous studies have reported the role of prophages, as a contributor to the Listeria diversity [56]. Diverse types of prophages found in the genome of Listeria lysogens could contribute to the variation of host genomes as mentioned in a previous study $[9,25]$.

\subsection{Change in the Genome Organization between Prophage and the Corresponding Induced Listeria Phage}

Prophages showed genome features with typical six gene clusters of temperate Listeria phages [17-20]. However, slight differences in the genome organization of prophage and the corresponding induced phage were observed. This observation could be explained by the phage packaging strategy. Induced phages LP019, LP040, and LP041 were predicted as circularly permuted, terminally redundant dsDNA phages [22], suggesting the opportunity for the phage genome to recombine both arms within the terminally redundant ends. As a result, the genome of each induced phage could become a circular dsDNA molecule before integrating into the host chromosome at an attachment site and becoming a prophage $[11,18]$. Furthermore, the additional sequence in prophage pp-134LM-comK or the altered nucleotide sequence in prophage pp-036LM-tRNA-Lys led to variations between prophage and the corresponding induced phage. This could be due to the gaining of accessory genes by either horizontal gene transfer by phage transduction $[70,71]$ or 
rapid niche-specific adaptation as proposed by Verghese et al. (2011) [11]. These findings are consistent with a previous report that alterations of Listeria genome during prophage induction or the acquisition of prophage genes is a main source of L. monocytogenes genome diversity [13].

\subsection{Uniqueness of Induced Phages and Temperate Listeria Phages from Various Sources and Regions}

Genomes of the induced phages from seafood and a seafood-related environment are closely related to the temperate phages from natural environments (specifically, wild mushroom, mushroom compost, and dairy farm) or induced phages from lysogens of other Listeria species. However, low sequence similarity was observed in the region of cell lysis and lysogeny control between the induced phages and the closely related temperate phages. In the cell lysis cluster, neither holin nor endolysin genes were conserved among the examined phages in this study. Similar to report of previous study by Dorscht et al. (2009), genes encoding for holin and endolysin showed a low similarity among the Listeria phages included in the analysis. These temperate and induced phages were not only from different sources, but also from distinct regions in the world, e.g., Thailand, Ireland, and the U.S.

The lysogeny control cluster of phages LP040 and LP041 showed high percentage (\%) similarity to their closely related temperate phages. This suggests the possibility that this phage may insert into the same genomic locus or have the same phage insertion site. This could be from the phage attachment site and the bacterial attachment site recombining between two DNA recognition sequences, resulting in the phage genome integrated into the bacterial chromosome by site-specific recombination [69,72]. This hypothesis was confirmed when we found that the induced phage LP041 and its closely related phage B054 was excised from the $3^{\prime}$ end of translation elongation factor gene, whereas the LP040 and the closely related phage A500 integrated into the tRNA-Lys [19]. However, the $\mathrm{Cro} / \mathrm{CI}$ repressor and anti-repressor genes involving in the phage genetic switch, which is responsible for expression of other phage genes leading to the launch of the lytic pathway or maintaining the lysogenic cycle [73], were found to be less conserved in all of the phages analyzed. These findings suggest a different potential mechanism required for prophage induction from the bacterial chromosome.

\section{Conclusions}

Here, we reported the draft genome sequences of two lysogenic isolates of L. monocytogenes (134LM and 036LM) obtained from seafood and a seafood-related environment. Intact prophages were found in the genomes of these two lysogens. A comparative prophage sequence analysis suggests that various prophage insertion sites, as well as diverse prophage sequences, could contribute to the diversity of Listeria in food processing environments. Overall, the study here provides a model for comparative analysis among prophages in diverse genomes of $L$. monocytogenes lysogens. Data from the detection of prophages, differentiation of insertion sites in specific loci of Listeria, and prophage sequence comparison together with the ANIb analysis, could further classify closely related L. monocytogenes strains/isolates. This study also suggests the potential development for using a prophage as a typing tool for monitoring and surveillance of foodborne pathogen contamination and transmission.

Supplementary Materials: The following are available online at https:/ /www.mdpi.com/article/10 .3390/microorganisms9071354/s1, Table S1: ANIb values of 2 L. monocytogenes lysogens sequenced in this study and 35 L. monocytogenes strains/ isolates sequenced previously and Figure S1: Linear genome visualization of the prophage sequences in each of the six identified clusters conducted using Easyfig 2.1.

Author Contributions: H.T.K.V.: experimental design, genome assembly, annotation and comparison, interpretation of data, produced tables/figures, and wrote the manuscript; M.J.S.: guide for genome analysis, interpretation of data, and edited the manuscript; S.B.: edited the manuscript; K.V.: 
conceived the study, guide for genome analysis, interpretation of data, and edited the manuscript. All authors approved the final manuscript. All authors have read and agreed to the published version of the manuscript.

Funding: This project was funded by the Prince of Songkla University (AGR600584S to KV); TRF Distinguished Research Professor Grant (to S.B). Funding from the Higher Education Research Promotion and Thailand's Education Hub for Southern Region of ASEAN Countries Project Office of the Higher Education Commission (to HTKV) are also acknowledged.

Institutional Review Board Statement: Not applicable.

Informed Consent Statement: Not applicable.

Acknowledgments: We acknowledge Varaporn Vuddhakul and the lab members at the Department of Microbiology, Prince of Songkla University, for their warm welcome, kindness, and helpful guidance during experimentation.

Conflicts of Interest: The authors declare that they have no competing interest.

\section{References}

1. Swaminathan, B.; Gerner-Smidt, P. The epidemiology of human listeriosis. Microbes Infect. 2007, 9, 1236-1243. [CrossRef] [PubMed]

2. Human Listeriosis. Available online: http://www.who.int/iris/handle/10665/62689 (accessed on 10 June 2016).

3. CDC Foodborne Outbreaks. Available online: http://www.cdc.gov/foodsafety/outbreaks/index.html (accessed on 12 December 2014).

4. CDC Foodborne Outbreaks. Available online: http://www.cdc.gov/listeria/outbreaks/index.html (accessed on 20 December 2015).

5. Tolvanen, R.; Hellström, S.; Elsser, D.; Morgenstern, H.; Björkroth, J.; Korkeala, H. Survival of Listeria monocytogenes strains in a dry sausage model. J. Food Prot. 2008, 71, 1550-1555. [CrossRef]

6. Burgess, C.M.; Gianotti, A.; Gruzdev, N.; Holah, J.; Knøchel, S.; Lehner, A.; Margas, E.; Esser, S.S.; Sela (Saldinger), S.; Tresse, O. The response of foodborne pathogens to osmotic and desiccation stresses in the food chain. Int. J. Food Microbiol. 2016, $221,37-53$. [CrossRef] [PubMed]

7. Palumbo, S.A.; Williams, A.C. Resistance of Listeria monocytogenes to freezing in foods. Food Microbiol. 1991, 8, 63-68. [CrossRef]

8. Tompkin, R.B. Control of Listeria monocytogenes in the food-processing environment. J. Food Prot. 2002, 65, 709-725. [CrossRef]

9. den Bakker, H.C.; Desjardins, C.A.; Griggs, A.D.; Peters, J.E.; Zeng, Q.; Young, S.K.; Kodira, C.D.; Yandava, C.; Hepburn, T.A.; Haas, B.J.; et al. Evolutionary dynamics of the accessory genome of Listeria monocytogenes. PLoS ONE 2013, 8, e67511. [CrossRef]

10. Ferreira, V.; Barbosa, J.; Stasiewicz, M.; Vongkamjan, K.; Moreno Switt, A.; Hogg, T.; Gibbs, P.; Teixeira, P.; Wiedmann, M. Diverse geno- and phenotypes of persistent Listeria monocytogenes isolates from fermented meat sausage production facilities in Portugal. Appl. Environ. Microbiol. 2011, 77, 2701-2715. [CrossRef] [PubMed]

11. Verghese, B.; Lok, M.; Wen, J.; Alessandria, V.; Chen, Y.; Kathariou, S.; Knabel, S. comK prophage junction fragments as markers for Listeria monocytogenes genotypes unique to individual meat and poultry processing plants and a model for rapid niche-specific adaptation, biofilm formation, and persistence. Appl. Environ. Microbiol. 2011, 77, 3279-3292. [CrossRef]

12. Orsi, R.H.; Borowsky, M.L.; Lauer, P.; Young, S.K.; Nusbaum, C.; Galagan, J.E.; Birren, B.W.; Ivy, R.A.; Sun, Q.; Graves, L.M.; et al. Short-term genome evolution of Listeria monocytogenes in a non-controlled environment. BMC Genom. 2008, 9, 539. [CrossRef] [PubMed]

13. Kuenne, C.; Billion, A.; Mraheil, M.A.; Strittmatter, A.; Daniel, R.; Goesmann, A.; Barbuddhe, S.; Hain, T.; Chakraborty, T. Reassessment of the Listeria monocytogenes pan-genome reveals dynamic integration hotspots and mobile genetic elements as major components of the accessory genome. BMC Genom. 2013, 14, 47. [CrossRef]

14. Nelson, K.E.; Fouts, D.E.; Mongodin, E.F.; Ravel, J.; DeBoy, R.T.; Kolonay, J.F.; Rasko, D.A.; Angiuoli, S.V.; Gill, S.R.; Paulsen, I.T.; et al. Whole genome comparisons of serotype $4 \mathrm{~b}$ and $1 / 2 \mathrm{a}$ strains of the food-borne pathogen Listeria monocytogenes reveal new insights into the core genome components of this species. Nucleic Acids Res. 2004, 32, 2386-2395. [CrossRef] [PubMed]

15. Chen, Y.; Luo, Y.; Curry, P.; Timme, R.; Melka, D.; Doyle, M.; Parish, M.; Hammack, T.S.; Allard, M.W.; Brown, E.W.; et al. Assessing the genome level diversity of Listeria monocytogenes from contaminated ice cream and environmental samples linked to a listeriosis outbreak in the United States. PLoS ONE 2017, 12, 1-19. [CrossRef] [PubMed]

16. Brüssow, H.; Canchaya, C.; Hardt, W.; Bru, H. Phages and the evolution of bacterial pathogens: From genomic rearrangements to lysogenic conversion. Microbiol. Mol. Biol. Rev. 2004, 68, 560-602. [CrossRef] [PubMed]

17. Zimmer, M.; Sattelberger, E.; Inman, R.B.; Calendar, R.; Loessner, M.J. Genome and proteome of Listeria monocytogenes phage PSA: An unusual case for programmed +1 translational frameshifting in structural protein synthesis. Mol. Microbiol. 2003, 50, 303-317. [CrossRef]

18. Loessner, M.J.; Inman, R.B.; Lauer, P.; Calendar, R. Complete nucleotide sequence, molecular analysis and genome structure of bacteriophage A118 of Listeria monocytogenes: Implications for phage evolution. Mol. Microbiol. 2000, 35, 324-340. [CrossRef] 
19. Dorscht, J.; Klumpp, J.; Bielmann, R.; Schmelcher, M.; Born, Y.; Zimmer, M.; Calendar, R.; Loessner, M.J. Comparative genome analysis of Listeria bacteriophages reveals extensive mosaicism, programmed translational frameshifting, and a novel prophage insertion site. J. Bacteriol. 2009, 191, 7206-7215. [CrossRef]

20. Denes, T.; Vongkamjan, K.; Ackermann, H.W.; Moreno Switt, A.I.; Wiedmann, M.; den Bakker, H.C. Comparative genomic and morphological analyses of Listeria phages isolated from farm environments. Appl. Environ. Microbiol. 2014, 80, 4616-4625. [CrossRef]

21. Casey, A.; Jordan, K.; Coffey, A.; McAuliffe, O. Complete genome sequences of vB_LmoS_188 and vB_LmoS_293, two bacteriophages with specificity for Listeria monocytogenes strains of serotypes 4b and 4e. Genome Announc. 2015, 3, e00040-15. [CrossRef] [PubMed]

22. Vu, H.T.K.; Stasiewicz, M.J.; Benjakul, S.; Vongkamjan, K. Genome sequences of Listeria phages induced from lysogenic isolates of Listeria monocytogenes from seafood and a seafood processing environment in Thailand. Genome Announc. 2018, 6, e00546-18. [CrossRef]

23. Vongkamjan, K.; Fuangpaiboon, J.; Turner, M.P.; Vuddhakul, V. Various ready-to-eat products from retail stores linked to occurrence of diverse Listeria monocytogenes and Listeria spp. isolates. J. Food Prot. 2016, 79, 239-245. [CrossRef]

24. Vongkamjan, K.; Fuangpaiboon, J.; Jirachotrapee, S.; Turner, M.P. Occurrence and diversity of Listeria spp. in seafood processing plant environments. Food Control 2015, 50, 265-272. [CrossRef]

25. Vu, H.T.K.; Benjakul, S.; Vongkamjan, K. Characterization of Listeria prophages in lysogenic isolates from foods and food processing environments. PLoS ONE 2019, 14, e0214641. [CrossRef] [PubMed]

26. Bolger, A.M.; Lohse, M.; Usadel, B. Trimmomatic: A flexible trimmer for Illumina sequence data. Bioinformatics 2014, 30, 2114-2120. [CrossRef]

27. Luo, R.; Liu, B.; Xie, Y.; Li, Z.; Huang, W.; Yuan, J.; He, G.; Chen, Y.; Pan, Q.; Liu, Y.; et al. SOAPdenovo2: An empirically improved memory-efficient short-read de novo assembler. Gigascience 2012, 1, 18. [CrossRef] [PubMed]

28. Darling, A.C.E.; Mau, B.; Blattner, F.R.; Perna, N.T. Mauve: Multiple alignment of conserved genomic sequence with rearrangements. Genome Res. 2004, 14, 1394-1403. [CrossRef]

29. Aziz, R.K.; Bartels, D.; Best, A.A.; DeJongh, M.; Disz, T.; Edwards, R.A.; Formsma, K.; Gerdes, S.; Glass, E.M.; Kubal, M.; et al. The RAST server: Rapid annotations using subsystems technology. BMC Genom. 2008, 9, 75. [CrossRef] [PubMed]

30. Lowe, T.M.; Chan, P.P. tRNAscan-SE On-line: Integrating search and context for analysis of transfer RNA genes. Nucleic Acids Res. 2016, 44, W54-W57. [CrossRef] [PubMed]

31. Lagesen, K.; Hallin, P.; Rødland, E.A.; Stærfeldt, H.-H.; Rognes, T.; Ussery, D.W. RNAmmer: Consistent and rapid annotation of ribosomal RNA genes. Nucleic Acids Res. 2007, 35, 3100-3108. [CrossRef]

32. Larsen, M.V.; Cosentino, S.; Rasmussen, S.; Friis, C.; Hasman, H.; Marvig, R.L.; Jelsbak, L.; Sicheritz-Ponten, T.; Ussery, D.W.; Aarestrup, F.M.; et al. Multilocus sequence typing of total-genome-sequenced bacteria. J. Clin. Microbiol. 2012, 50, $1355-1361$. [CrossRef]

33. Jolley, K.A.; Maiden, M.C.J. BIGSdb: Scalable analysis of bacterial genome variation at the population level. BMC Bioinform. 2010, 11, 595. [CrossRef]

34. Pightling, A.W.; Petronella, N.; Pagotto, F. The Listeria monocytogenes Core-Genome Sequence Typer (LmCGST): A bioinformatic pipeline for molecular characterization with next-generation sequence data. BMC Microbiol. 2015, 15. [CrossRef] [PubMed]

35. Hyden, P.; Pietzka, A.; Lennkh, A.; Murer, A.; Springer, B.; Blaschitz, M.; Indra, A.; Huhulescu, S.; Allerberger, F.; Ruppitsch, W.; et al. Whole genome sequence-based serogrouping of Listeria monocytogenes isolates. J. Biotechnol. 2016, 235, 181-186. [CrossRef] [PubMed]

36. Richter, M.; Rosselló-Móra, R.; Oliver Glöckner, F.; Peplies, J. JSpeciesWS: A web server for prokaryotic species circumscription based on pairwise genome comparison. Bioinformatics 2016, 32, 929-931. [CrossRef] [PubMed]

37. Arndt, D.; Grant, J.R.; Marcu, A.; Sajed, T.; Pon, A.; Liang, Y.; Wishart, D.S. PHASTER: A better, faster version of the PHAST phage search tool. Nucleic Acids Res. 2016, 44, W16-W21. [CrossRef] [PubMed]

38. Altschul, S.F.; Gish, W.; Miller, W.; Myers, E.W.; Lipman, D.J. Basic local alignment search tool. J. Mol. Biol. 1990, 215 , 403-410. [CrossRef]

39. Rutherford, K.; Parkhill, J.; Crook, J.; Horsnell, T.; Rice, P.; Rajandream, M.-A.; Barrell, B. Artemis: Sequence visualization and annotation. Bioinformatics 2000, 16, 944-945. [CrossRef]

40. Quevillon, E.; Silventoinen, V.; Pillai, S.; Harte, N.; Mulder, N.; Apweiler, R.; Lopez, R. InterProScan: Protein domains identifier. Nucleic Acids Res. 2005, 33, W116-W120. [CrossRef]

41. Ragon, M.; Wirth, T.; Hollandt, F.; Lavenir, R.; Lecuit, M.; Monnier, A.L.; Brisse, S. A new perspective on Listeria monocytogenes evolution. PLoS Pathog. 2008, 4. [CrossRef]

42. Chenal-francisque, V.; Lopez, J.; Cantinelli, T.; Caro, V.; Tran, C.; Leclercq, A.; Lecuit, M.; Brisse, S. Worldwide distribution of major clones of Listeria monocytogenes. Emerg. Infect. Dis. 2011, 17, 1110-1112. [CrossRef]

43. Wang, Y.; Zhao, A.; Zhu, R.; Lan, R.; Jin, D.; Cui, Z.; Wang, Y.; Li, Z.; Wang, Y.; Xu, J.; et al. Genetic diversity and molecular typing of Listeria monocytogenes in China. BMC Microbiol. 2012, 12, 119. [CrossRef]

44. Schmitz-Esser, S.; Müller, A.; Stessl, B.; Wagner, M. Genomes of sequence type 121 Listeria monocytogenes strains harbor highly conserved plasmids and prophages. Front. Microbiol. 2015, 6, 380. [CrossRef] [PubMed] 
45. Meier-Kolthoff, J.P.; Göker, M. VICTOR: Genome-based phylogeny and classification of prokaryotic viruses. Bioinformatics 2017, 33, 3396-3404. [CrossRef] [PubMed]

46. FigTree 1.4.3-A Graphical Viewer of Phylogenetic Trees and a Program for Producing Publication-Ready Figures. Available online: http:/ / tree.bio.ed.ac.uk/software/figtree/ (accessed on 12 June 2018).

47. Sullivan, M.J.; Petty, N.K.; Beatson, S.A. Easyfig: A genome comparison visualizer. Bioinformatics 2011, 27, 1009-1010. [CrossRef]

48. Sievers, F.; Wilm, A.; Dineen, D.; Gibson, T.J.; Karplus, K.; Li, W.; Lopez, R.; McWilliam, H.; Remmert, M.; Soding, J.; et al. Fast, scalable generation of high-quality protein multiple sequence alignments using Clustal Omega. Mol. Syst. Biol. 2014, 7, 539. [CrossRef] [PubMed]

49. Chen, Y.; Zhang, W.; Knabel, S.J. Multi-virulence-locus sequence typing identifies single nucleotide polymorphisms which differentiate epidemic clones and outbreak strains of Listeria monocytogenes. J. Clin. Microbiol. 2007, 45, 835-846. [CrossRef]

50. Kruczkiewicz, P.A. Comparative Genomic Framework for the In Silico Design and Assessment of Molecular Typing Methods Using Whole-Genome Sequence Data with Application to Listeria Monocytogenes. Master's of Science Thesis, University of Lethbridge, Lethbridge, AB, Canada, 2013.

51. Reimer, A.; Weedmark, K.; Petkau, A.; Peterson, C.-L.; Walker, M.; Knox, N.; Kent, H.; Mabon, P.; Berry, C.; Tyler, S.; et al. Shared genome analyses of notable listeriosis outbreaks, highlighting the critical importance of epidemiological evidence, input datasets and interpretation criteria. Microb. Genom. 2019, 5. [CrossRef] [PubMed]

52. Ziegler, J. The distribution, diversity and functional characterization of the Listeria genomic island 1. Master's of Science Thesis, University of Manitoba, Winnipeg, MB, Canada, 2011.

53. Briers, Y.; Klumpp, J.; Schuppler, M.; Loessner, M.J. Genome sequence of Listeria monocytogenes Scott A, a clinical isolate from a food-borne listeriosis outbreak. J. Bacteriol. 2011, 193, 4284-4285. [CrossRef]

54. Chen, Y.; Luo, Y.; Carleton, H.; Timme, R.; Melka, D.; Muruvanda, T.; Wang, C.; Kastanis, G.; Katz, L.S.; Turner, L.; et al. Whole genome and core genome multilocus sequence typing and single nucleotide polymorphism analyses of Listeria monocytogenes associated with an outbreak linked to cheese, United States, 2013. Appl. Environ. Microbiol. 2017, 83, e00633-17. [CrossRef] [PubMed]

55. Cherifi, T.; Carrillo, C.; Lambert, D.; Miniaï, I.; Quessy, S.; Larivière-Gauthier, G.; Blais, B.; Fravalo, P. Genomic characterization of Listeria monocytogenes isolates reveals that their persistence in a pig slaughterhouse is linked to the presence of benzalkonium chloride resistance genes. BMC Microbiol. 2018, 18, 1-13. [CrossRef]

56. Matle, I.; Pierneef, R.; Mbatha, K.R.; Magwedere, K.; Madoroba, E. Genomic diversity of common sequence types of Listeria monocytogenes isolated from ready-to-eat products of animal origin in South Africa. Genes (Basel) 2019, 10, 1007. [CrossRef]

57. Burall, L.S.; Grim, C.J.; Mammel, M.K.; Datta, A.R. Whole genome sequence analysis using JSpecies tool establishes clonal relationships between Listeria monocytogenes strains from epidemiologically unrelated listeriosis outbreaks. PLoS ONE 2016, 11, e0150797. [CrossRef]

58. Schmitz-Esser, S.; Gram, L.; Wagner, M. Complete genome sequence of the persistent Listeria monocytogenes strain R479a. Genome Announc. 2015, 3, e00150-15. [CrossRef] [PubMed]

59. Glaser, P.; Frangeul, L.; Buchrieser, C.; Rusniok, C.; Amend, A.; Baquero, F.; Berche, P.; Bloecker, H.; Brandt, P.; Chakraborty, T.; et al. Comparative genomics of Listeria species. Science 2001, 294, 849-852. [CrossRef]

60. Hain, T.; Ghai, R.; Billion, A.; Kuenne, C.; Steinweg, C.; Izar, B.; Mohamed, W.; Mraheil, M.; Domann, E.; Schaffrath, S.; et al. Comparative genomics and transcriptomics of lineages I, II, and III strains of Listeria monocytogenes. BMC Genom. 2012, 13, 144. [CrossRef]

61. Steele, C.L.; Donaldson, J.R.; Paul, D.; Banes, M.M.; Arick, T.; Bridges, S.M.; Lawrence, M.L. Genome sequence of lineage III Listeria monocytogenes strain HCC23. J. Bacteriol. 2011, 193, 3679-3680. [CrossRef]

62. Chen, J.; Xia, Y.; Cheng, C.; Fang, C.; Shan, Y.; Jin, G.; Fang, W. Genome sequence of the nonpathogenic Listeria monocytogenes serovar 4a strain M7. J. Bacteriol. 2011, 193, 5019-5020. [CrossRef] [PubMed]

63. Bailey, T.W.; do Nascimento, N.C.; Bhunia, A.K. Genome sequence of Listeria monocytogenes strain F4244, a 4b serotype. Genome Announc. 2017, 5, 1-2. [CrossRef] [PubMed]

64. Gilmour, M.W.; Graham, M.; Van Domselaar, G.; Tyler, S.; Kent, H.; Trout-Yakel, K.M.; Larios, O.; Allen, V.; Lee, B.; Nadon, C. High-throughput genome sequencing of two Listreia monocytogenes clinical isolates during a large foodborne outbreak. BMC Genom. 2010, 11, 120. [CrossRef]

65. Zink, R.; Loessner, M.J.; Scherer, S. Characterization of cryptic prophages (monocins) in Listeria and sequence analysis of a holin/endolysin gene. Microbiology 1995, 141, 2577-2584. [CrossRef] [PubMed]

66. Rychli, K.; Wagner, E.M.; Ciolacu, L.; Zaiser, A.; Tasara, T.; Wagner, M.; Schmitz-Esser, S. Comparative genomics of human and non-human Listeria monocytogenes sequence type 121 strains. PLoS ONE 2017, 12, e0176857. [CrossRef]

67. Rabinovich, L.; Sigal, N.; Borovok, I.; Nir-Paz, R.; Herskovits, A.A. Prophage excision activates Listeria competence genes that promote phagosomal escape and virulence. Cell 2012, 150, 792-802. [CrossRef]

68. Fox, E.M.; Allnutt, T.; Bradbury, M.I.; Fanning, S.; Chandry, P.S. Comparative genomics of the Listeria monocytogenes ST204 subgroup. Front. Microbiol. 2016, 7, 1-12. [CrossRef] [PubMed]

69. Julien, B. Characterization of the integrase gene and attachment site for the Myxococcus xanthus Bacteriophage Mx9. J. Bacteriol. 2003, 185, 6325-6330. [CrossRef] 
70. Fineran, P.C.; Petty, N.K.; Salmond, G.P.C. Transduction: Host DNA transfer by bacteriophages. In The Encyclopedia of microbiology; Schaechter, M., Ed.; Academic Press: London, UK, 2009; pp. 666-679, ISBN 9780123739445.

71. Eggers, C.H.; Gray, C.M.; Preisig, A.M.; Glenn, D.M.; Pereira, J.; Ayers, R.W.; Alshahrani, M.; Acabbo, C.; Becker, M.R.; Bruenn, K.N.; et al. Phage-mediated horizontal gene transfer of both prophage and heterologous DNA by $\phi$ BB-1, a bacteriophage of Borrelia burgdorferi. Pathog. Dis. 2016, 74, ftw107. [CrossRef] [PubMed]

72. Groth, A.C.; Calos, M.P. Phage integrases: Biology and applications. J. Mol. Biol. 2004, 335, 667-678. [CrossRef] [PubMed]

73. Gandon, S. Why be temperate: Lessons from bacteriophage $\lambda$. Trends Microbiol. 2016, 24, 356-365. [CrossRef] 